\title{
NOUVELle
}

\section{Le neuropeptide FF, aiguilleur des macrophages du tissu adipeux}

Raphaëlle Quillet ${ }^{1}$, Frédéric Simonin ${ }^{1}$

\author{
${ }^{1}$ Biotechnologie et signalisation cellulaire, \\ CNRS UMR 7242, université de Strasbourg, \\ 300, boulevard Sébastien Brant, 67412 IIlkirch, France. \\ simonin@unistra.fr
}

$>$ Les chiffres de l'Organisation mondiale de la santé concernant l'obésité sont alarmants. Depuis plus de 30 ans, cette pathologie est apparue progressivement au sein de notre société, favorisée par des régimes caloriques et composés de lipides en excès. En 2014, $13 \%$ de la population mondiale étaient considérés comme obèses, et le développement des maladies métaboliques et cardiovasculaires associées n'a pas cessé de croître. L'obésité est une maladie du tissu adipeux. Ce tissu est constitué d'adipocytes, dont le rôle est de stocker des réserves énergétiques, mais également de cellules du système immunitaire, appelées macrophages du tissus adipeux (ATM pour adipose tissue macrophage) [1] $(\rightarrow)$.

$\rightarrow$ Voir la Synthèse de $\varepsilon$. Delmas et al., $m / s n^{\circ} 11$, novembre 211, page 993

Les cellules immunitaires du tissu adipeux jouent un rôle majeur dans le développement et le métabolisme de ce tissu [2]. En cas d'obésité, les macrophages s'y accumulent et se différencient en un type spécifique, appelé Ml (pour macrophage de type 1 , en référence aux lymphocytes Th[helper] 1), caractérisé par un fort potentiel pro-inflammatoire [3]. L'inflammation, qui est liée à l'infiltration du tissu adipeux par ces macrophages, est l'une des altérations majeures que l'on observe chez les patients souffrant d'obésité. Elle est responsable des maladies métaboliques concomitantes à l'évolution de la pathologie, comme le diabète de type 2 et les dyslipidémies. Les macrophages de type 1 produisent des cytokines inflammatoires, de l'oxyde d'azote (NO) et des espèces réactives de l'oxygène (ROS) qui sont à l'origine de l'inflammation du tissu et conduisent à une résistance à I'insuline $[4,5]$. In vitro, les macrophages Ml sont activés par différents traitements comme le LPS (lipopolysaccharide) bactérien, l'interféron- $\gamma$ (IFN$\gamma$ ), ou l'acide palmitique.

Les macrophages du tissu adipeux, ATM, peuvent également adopter un état d'activation de type 2, ou M2. Par leur action anti-inflammatoire, ces macrophages M2 sont capables de limiter l'inflammation et la résistance à l'insuline. Cet état, qui est bénéfique d'un point de vue métabolique, est donc favorable. II peut être induit par différentes molécules, parmi lesquelles les cytokines de type Th2 ( $T$ helper 2), les métabolites lipidiques, les neurotransmetteurs ou encore les hormones [4]. Les mécanismes impliqués dans cette transition d'état d'activation des macrophages restent cependant encore inconnus.

Le neuropeptide FF (NPFF) est un membre de la famille des peptides RF-amides ${ }^{1}$. Il a été principalement étudié chez les mammifères pour son rôle modulateur de la douleur dans les réponses adaptatives aux traitements par les opiacés. Certains éléments indiquent qu'il pourrait également être impliqué dans la réduction de la prise alimentaire chez les rongeurs, via une action hypothalamique [6]. Des études menées in vitro ont montré qu'il inhibait le développement des adipocytes chez la souris et l'Homme [7]. Le récepteur du NPFF, NPFFR2 (NPFF recep-

\footnotetext{
${ }^{1}$ La famille des peptides RF-amides regroupe des neuropeptides qui partagent la même séquence $\mathrm{N}$-terminale à savoir $\operatorname{Arg}(\mathrm{R})-\mathrm{Phe}(\mathrm{F})-\mathrm{NH}_{2}$.
}

tor 2), est très faiblement exprimé dans les tissus périphériques, à l'exception du tissu adipeux $[8,9]$. Dans ce tissu, NPFF serait libéré à partir des terminaisons nerveuses [9], mais son action sur les adipocytes reste inconnue. Des variants génétiques du récepteur NPFFR2 ont cependant été associés à l'obésité, ce qui renforce l'hypothèse de l'existence d'un lien entre NPFF et cette maladie [10].

Le NPFF est présent dans la circulation sanguine, en concentration suffisante pour activer les ATM en macrophages M2

L'étude que nous avons menée au sein du consortium international coordonné par le Dr Tamas Röszer a été publiée récemment dans le Journal of Clinical Investigation [11]. Dans cet article, nous avons montré que le neuropeptide FF (NPFF) avait un effet bénéfique sur le métabolisme: il amplifie en effet, chez l'Homme et la souris, l'activation des macrophages de type 2 (M2) et leur prolifération, ce qui permet le maintien d'un tissu adipeux sain (Figure 1). Chez la souris, nous avons montré que le NPFF se liait à ses récepteurs présents à la surface des macrophages du tissu adipeux (ATM) avec une constante de dissociation $\left(K_{D}\right)$ de $0,37 \mathrm{nM}$, et qu'il induisait une diminution de l'accumulation d'AMP cyclique en activant la sous-unité inhibitrice de l'adénylate cyclase $G_{i}$. Nous avons aussi montré que sa concentration, dans les plasmas murin et humain, était compatible avec son action sur son récepteur. Elle est diminuée en cas d'obésité, mais une restriction calorique permet d'inverser cette situation. 


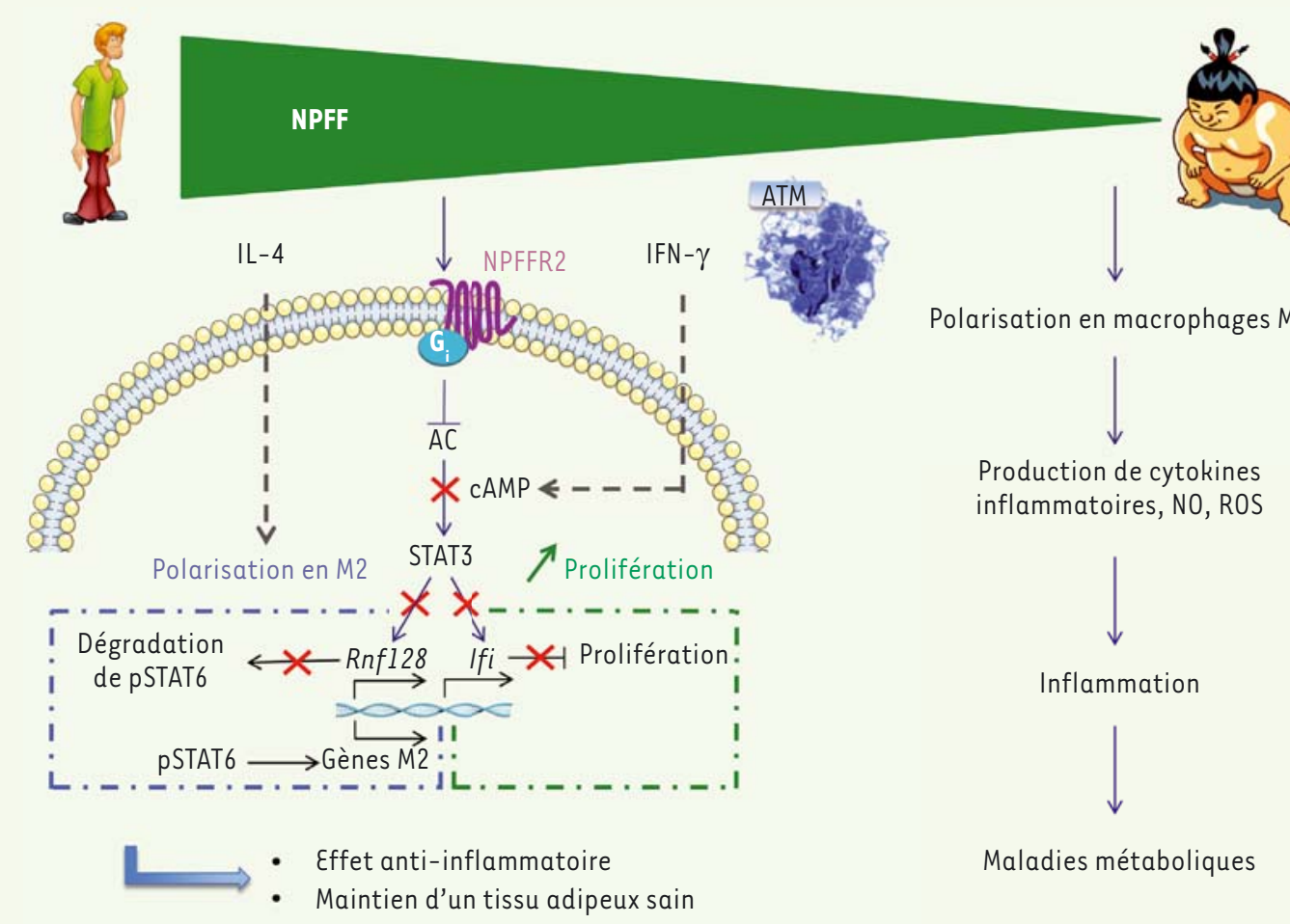

Figure 1. Le système NPFF/NPFFR2 inhibe la signalisation IFN- $\gamma$ et empêche l'inflammation du tissu adipeux. La liaison du NPFF au récepteur NPFFR2 bloque l'adénylate cyclase (AC) et, ainsi, la production d'adénosine monophosphate cyclique (AMPc), ce qui inhibe la voie dépendante de STAT3 (signal transducer and activator of transcription 3). Une diminution de la transcription de Rnf128 codant une $\varepsilon 3$-ubiquitine ligase retarde la dégradation de phospho-STAT6 et permet la transcription des gènes induisant un phénotype M2. L'inhibition des gènes inductibles par l'IFN- $\gamma$ (interféron-gamma) favorise la prolifération des macrophages. Cette cascade conduit à l'action anti-inflammatoire du NPFF et au maintien d'un tissu adipeux sain. En revanche, le faible taux de NPFF retrouvé chez les patients obèses entrâne la polarisation des macrophages en macrophages de type Ml, qui produisent des cytokines inflammatoires, de l'oxyde nitrique (NO) et des espèces réactives de l'oxygène (ROS). L'inflammation des tissus qui en résulte peut conduire à l'apparition de maladies métaboliques et contribuer aux comorbidités associées à l'obésité.

Le NPFF stimule, chez la souris, la transcription de gènes qui codent des molécules impliquées dans les fonctions des macrophages M2, comme AGMO (alkylglycérol monooxygénase, connue pour son potentiel anti-inflammatoire), le récepteur $\alpha$ de l'IL-4 (interleukin-4 receptor, IL4Ra), I'Argl (arginase-1) et l'IL-10. Chez l'Homme, il induit également la transcription de I'IL4Ra et de AGMO mais aussi du macrophage mannose receptor (MR ou CD206). Ces activations transcriptionnelles, spécifiques des macrophages M2, coïncident avec la diminution de l'expression des marqueurs caractéristiques des macrophages $\mathrm{Ml}$ : I'IL-6 et le TNF- $\alpha$ (tumor necrosis factoralpha). Le traitement des ATM murins et humains par le NPFF mime ainsi l'effet de l'IL-4 en favorisant l'orientation M2. Cependant, le traitement des ATM murins, avec une dose de NPFF correspondant à la concentration plasmatique trouvée chez des patients obèses, ne permet pas d'observer d'activation des macrophages en macrophages de type M2. Les taux plasmatiques de NPFF qui sont détectés chez ces patients sont donc insuffisants pour activer les macrophages. À noter que les ATM de souris soumises à un régime riche en graisses durant 12 semaines (correspondant à un contexte d'obésité) ont des taux de récepteurs NPFFR2 qui sont diminués par rapport à ceux des souris nourries sans excès. Ceci suggère que le défaut d'orientation en macrophages M2 aurait pour origine un déficit du système NPFF.
Le NPFF inhibe la dégradation de phospho-STAT6 dans les ATM

De même que I'IL-4 active les macrophages par l'intermédiaire de STAT6 (signal transducer and activator of transcription-6) [12], la transition d'état induite par le NPFF dépend de la voie de signalisation impliquant STAT6. L'expression des gènes activés par l'IL-4 et qui sont impliqués dans l'activation des macrophages anti-inflammatoires (de type M2) est également observée après stimulation par le NPFF (Figure 1). En particulier, la transcription de RNF128, qui code une ¿3 ubiquitine-ligase responsable de la dégradation de la forme phosphorylée de STAT6 (phospho-STAT6), est diminuée par le NPFF comme par l'IL-4. La réduction de RNF128, dans les ATM traités avec le NPFF, apparaît donc responsable du maintien 
de la phosphorylation de STAT6. Dans des cellules macrophagiques de souris J774A.1 surexprimant RNF128, le NPFF est incapable d'induire l'orientation des cellules en macrophages $M 2$. En revanche, des ATM de souris déficientes en RFN128 présentent des taux élevés de phospho-STAT6 et expriment fortement les marqueurs d'activation de cellules M2 (comme Arg-1 et CD206), à l'instar de cellules surexprimant le récepteur NPFFR2. Le taux de RNF128 dans les ATM humains, et chez les souris nourries avec un régime riche en graisse, diminue après traitement avec le NPFF. Ces effets ne sont pas retrouvés chez des souris génétiquement modifiées déficientes en NPFFR2, ce qui confirme son rôle dans la polarisation des macrophages et la spécificité de la cible d'action du NPFF.

\section{Le NPFF favorise l'auto- renouvellement des ATM}

Nos études démontrent que le NPFF induit la prolifération des macrophages du tissu adipeux chez la souris et l'Homme. Cet effet n'est pas observé pour les ATM provenant de souris déficientes en NPFFR2 ou dans des expériences de blocage pharmacologique du récepteur. Le NPFF réduit en effet les niveaux de transcription de Mafb (musculoaponeurotic fibrosarcoma bZIP transcription factor $B$ ) et ceux des gènes codant la famille des IFI200 (IFN-inducible 200), des régulateurs négatifs de la prolifération des macrophages. En revanche, le traitement par le NPFF, ou la surexpression du récepteur NPFFR2, augmente la transcription de Ndrg2 ( $N$-myc downstreamregulated gene 2) qui code un facteur impliqué dans la prolifération de différents types cellulaires.

NPFF inhibe la signalisation IFN- $\boldsymbol{\gamma}$, une clé pour l'activation et la prolifération des ATM

L'interféron- $\gamma($ IFN- $\gamma)$ est à l'origine d'une diminution de la transcription de NPFFR2. II active des facteurs pro-inflammatoires comme l'IL-6 et la Nos2 (nitric oxide synthase 2) dans les ATM. À l'inverse, le NPFF inhibe la Nos2 et la transcription de gènes inductibles par l'IFN- $\gamma$ dans la lignée
J774A.1. À noter que le traitement par I'IFN- $\gamma$ induit des effets opposés à ceux du NPFF sur les gènes qu'il régule, comme RNF128, Ndrg2 et les membres de la famille IFI200. Ces effets antagonistes entre IFN- $\gamma$ et NPFF s'observent également sur la production d'AMP cyclique intracellulaire, qui diminue sous l'action du NPFF, ce qui conduit à la polarisation des macrophages en M2 et à la prolifération des ATM.

\section{Conclusion}

Notre étude révèle que le NPFF est un puissant régulateur de la qualité et de la quantité des macrophages résidents du tissu adipeux. Dans les macrophages, la liaison de NPFF à son récepteur, NPFFR2, active la voie de signalisation dépendant de STAT6, qui stimule leur polarisation en macrophages de type $M 2$, et provoque leur auto-renouvellement. Actuellement, les effets du NPFF sur le métabolisme et, notamment, sur le contrôle de l'appétit et la thermogenèse, sont classiquement associés à ses fonctions de neurotransmetteur dans le système nerveux central. Notre étude démontre, pour la première fois, que ce neuropeptide a également de puissants effets périphériques, en agissant comme une hormone, et que le pancréas est un site d'expression du gène codant le NPFF, Npff. Une diminution de sa traduction pourrait ainsi être à l'origine de la réduction du taux plasmatique de NPFF observée chez la personne obèse. L'expression de NPFF et de son récepteur, par les macrophages du tissu adipeux, est réduite chez les patients obèses, en comparaison à celle des individus sains. Cela pourrait conduire à la perte de l'effet du peptide sur les macrophages, ce qui est en accord avec le paradigme actuel décrivant une relation entre obésité et dérégulation de l'activation et de la prolifération des ATM (Figure 1). Chez les rongeurs, le NPFF est un facteur anorexigène. II agit en effet sur les noyaux hypothalamiques. Or, la balance entre les signaux orexigènes et anorexigènes dans les centres de l'hypothalamus contrôlant l'appétit est une clé dans le développement de l'obésité. $\varepsilon n$ conclusion, cette étude montre que le NPFF contrôle l'auto-renouvellement des macrophages du tissu adipeux et leur polarisation en macrophages M2. II protège de l'inflammation du tissu adipeux, principal facteur de prédisposition au syndrome de résistance à l'insuline. Le NPFF et son récepteur NPFFR2 représentent donc des cibles thérapeutiques potentielles et prometteuses pour les futurs traitements des défauts de régulation de la prolifération et de l'activation des ATM associés à l'obésité. $\diamond$

The neuropeptide FF: a signal for $M \mathbb{1}$ to $M 2$ type switching in macrophages from the adipose tissue

\section{LIENS D'INTÉRÊT}

Les auteurs déclarent n'avoir aucun lien d'intérêt concernant les données publiées dans cet article.

\section{RÉFÉRENCES}

1. Delmas $\varepsilon$, Tordjman J, Guerre-Millo, Clément K. Le tissu adipeux : un nouveau terrain de jeu pour les cellules immunitaires. Med Sci (Paris) 2011 ; 27 : 993-9.

2. Boutens L, Stienstra R. Adipose tissue macrophages: going off track during obesity. Diabetologia 2016; $59: 879-94$.

3. Amano SU, Cohen JL, Vangala P, et al. Local proliferation of macrophages contributes to obesityassociated adipose tissue inflammation. Cell Metab 2014 ; 19 : 162-71.

4. Roszer T. Understanding the mysterious M2 macrophage through activation markers and effector mechanisms. Mediators Inflamm 2015; 2015 : 816460.

5. Rosen $E D$, Spiegelman BM. What we talk about when we talk about fat. Cell $2014 ; 156: 20-44$.

6. Quillet R, Ayachi S, Bihel F, et al. RF-amide neuropeptides and their receptors in mammals: Pharmacological properties, drug development and main physiological functions. Pharmacol Ther 2016 ; $160: 84-132$

7. Herrera-Herrera ML, Salazar-Olivo LA. RFamide neuropeptides inhibit murine and human adipose differentiation. Biochem Biophys Res Commun 2008 ; $377: 29-34$.

8. Bonini JA, Jones KA, Adham N, et al. Identification and characterization of two $G$ protein-coupled receptors for neuropeptide FF. J Biol Chem 2000 ; 275 : 39324-31.

9. van Harmelen V, Dicker A, Sjolin $\varepsilon$, et al. Effects of pain controlling neuropeptides on human fat cell lipolysis. Int J Obes (Lond) 2010 ; 34 : 1333-40.

10. Hunt SC, Hasstedt SJ, Xin Y, et al. Polymorphisms in the NPY2R gene show significant associations with BMI that are additive to FTO, MC4R, and NPFFR2 gene effects. Obesity (Silver Spring) $2011 ; 19: 2241-7$.

11. Waqas SFH, Hoang AC, Lin YT, et al. Neuropeptide FF increases $\mathrm{M} 2$ activation and self-renewal of adipose tissue macrophages. J Clin Invest 2017 ; 127 : 2842-54.

12. Lawrence $T$, Natoli $G$. Transcriptional regulation of macrophage polarization: enabling diversity with identity. Nat Rev Immunol 2011 ; 11 : 750-61. 